\section{Generating correlation matrices with model error for simulation studies in factor analysis: A combination of the Tucker-Koopman-Linn model and Wijsman's algorithm}

\author{
SEHEE HONG \\ University of California, Santa Barbara, Califormia
}

Most simulation studies in factor analysis follow a process of constructing population correlation matrices from the common-factor model and generating sample correlation matrices from the population matrices. In the common-factor model, the population correlation matrix is perfectly fit by the model's containing common and unique factors. However, since no mathematical model accounts exactly for the real-world phenomena that it is intended to represent, the Tucker-Koopman-Linn model (1969) is more realistic for generating correlation matrices than the conventional common-factor model because the former incorporates model error. In this paper, a procedure for generating population and sample correlation matrices with model error by combining the Tucker-Koopman-Linn model and Wijsman's algorithm (1959) is presented. The SAS/IML program for generating correlation matrices is described, and an example is also provided.

There has been an increase in the number of simulation studies in factor analysis over the past decade. Most studies have followed a process of constructing population correlation matrices from the common-factor model and generating sample correlation matrices from the population matrices. The common-factor model can be expressed as follows:

$$
P=\Lambda \Phi \Lambda+D,
$$

where $P$ is a $k \times k$ population correlation matrix for $k$ measured variables (assuming that measured variables are standardized), $\Lambda$ is a $k \times r$ factor loading matrix for $r$ common factors, $\Phi$ is an $r \times r$ matrix of correlations among common factors, and $D$ is a $k \times k$ diagonal matrix of unique variances. In this representation of the model, the population matrix for the measured variables is perfectly fit by the model's containing $r$ common factors and $k$ unique factors.

However, as several researchers argue (e.g., Cudeck \& Henly, 1991; MacCallum \& Tucker, 1991; McDonald, 1989), models are simplifications of underlying processes

I am grateful to Roger Millsap for his help with developing the Tucker-Koopman-Linn model that includes correlations among major and minor factors. Special thanks are extended to Rebecca Zwick for commenting on an earlier version of this article. Correspondence regarding this article should be addressed to $\mathrm{S}$. Hong, Department of Education, University of California, Santa Barbara, CA 93106 (e-mail: shong@education.ucsb.edu). and, therefore, no mathematical model accounts exactly for the real-world phenomena that it is intended to represent. From this point of view, the representation of the model is inappropriate in that it does not explicitly provide for the lack of fit (i.e., model error) between the model and the real-world phenomena. One of the main sources of the lack of fit is the existence of large numbers of "unmodeled" minor common factors (MacCallum \& Tucker, 1991). If results from simulation studies can be generalized to the extent that the simulation procedures produce realistic data, generalizability of simulation studies that use "unrealistic" data generated from the common-factor model may be problematic.

A more realistic model that incorporates minor factors was proposed by Tucker, Koopman, and Linn (1969). The mathematical model is

$$
P=V V^{\prime}+D+W W^{\prime},
$$

where the population correlation matrix $P$ of order $k$ can be generated on the basis of the three matrices: $V(k \times r$ major factor loading matrix $), D(k \times k$ diagonal matrix of unique factor variances $)$, and $W(k \times q$ minor factor loading matrix).

Although Tucker et al. (1969) did not provide the method for including correlations among major and minor factors in the model, it is possible to incorporate factor correlations. Let $J=\left[\begin{array}{ll}V & W\end{array}\right]$ be the super loading matrix with columns made up of $V$ and $W$, and $B$ be the matrix of factor correlations. Then, $J$ is a $k \times(r+q)$ matrix and $B$ is an $(r+q) \times(r+q)$ matrix. The $B$ matrix can be partitioned as follows:

$$
B=\left[\begin{array}{cc}
\Phi & \Upsilon \\
\Upsilon^{\prime} & \Gamma
\end{array}\right],
$$

where $\Phi$ is an $r \times r$ matrix of correlations among major factors and $\Gamma$ is a $q \times q$ matrix of correlations among minor factors. $Y$ is an $r \times q$ matrix of correlations among major and minor factors. Then, the population correlation matrix $P$ is

$$
\mathrm{P}=J B J^{\prime}+D .
$$

Note that $J B J^{\prime}+D$ becomes $V V^{\prime}+D+W W^{\prime}$, when both $\Phi$ and $\Gamma$ are identity matrices and $Y$ is a null matrix. With the model in Equation 4, it is possible to generate population correlation matrices with model error. A demonstration of generating population matrices is given in the Numerical Example section.

Once the population correlation matrix $P$ is derived with the Tucker-Koopman-Linn procedure (1969), sample correlation matrices can be generated from the population. Kaiser and Dickman's (1962) algorithm has been used for years for generating sample matrices (e.g., MacCallum, 1986; Silvia \& MacCallum, 1988). Given a population correlation matrix $P$, the Kaiser and Dickman pro- 
Table 1

Parameters for Generating Population Correlation Matrix, $P$

$\begin{aligned} V & =\left[\begin{array}{rrr}.673 & .020 & -.039 \\ .606 & .136 & .008 \\ .711 & .012 & .068 \\ .638 & -.085 & -.007 \\ -.058 & .529 & -.019 \\ .008 & .727 & .061 \\ .035 & .609 & -.053 \\ .197 & .618 & .174 \\ -.002 & -.002 & .601 \\ -.003 & .001 & .626\end{array}\right] \\ \text { Minor-factor variances } & =\left[\begin{array}{r}.100 \\ .100 \\ .100 \\ .100 \\ .100 \\ .100 \\ .100 \\ .100 \\ .100 \\ .100\end{array}\right]\end{aligned}$

cedure is based on the fundamental postulate of components analysis,

$$
Z=X F^{\prime} \text {, }
$$

where $X$ is an $n \times k$ matrix whose elements are randomly generated from a normal distribution with mean 0 and variance 1 , and $F$ is a $k \times k$ principal components matrix of $P$. Here, $F$ is computed as follows:

$$
F=U D^{\frac{1}{2}}
$$

where $U$ is a matrix with columns formed from the $k$ eigenvectors of $P$ and $D$ is a diagonal matrix with the corresponding $k$ eigenvalues as diagonal elements. Then, the resulting $Z$ is an $n \times k$ matrix that contains observations from a multivariate normal population with zero means and unit standard deviations. The sample correlation matrix is generated by computing correlations among $k$ variables with the $n$ observations in the $Z$ matrix. Fron a population correlation matrix, sample correlation matrices can be generated.

A drawback of the Kaiser and Dickman (1962) procedure is that the $X$ matrix must be generated to produce sample correlation matrices, which is computationally intensive particularly for large sample sizes. An alternative method proposed by Wijsman (1959) can reduce computing cost by generating sample correlation matrices without producing the $X$ matrix. Therefore, it is more computationally efficient than Kaiser and Dickman's procedure. Given the population matrix of order $k$, the Wijsman procedure starts by generating the matrix $A$ as follows:

$$
A=F G G^{\prime} F^{\prime} .
$$

In this case, $F$ is a $k \times k$ factor matrix of $P$. A factoring can be obtained such that

$$
P=F F^{\prime} .
$$

In Equation 7, the $G$ matrix is a lower triangular $k \times k$ matrix, randomly generated. Its off-diagonal entries are random normal deviates, drawn from a normal distribution with mean 0 and variance 1 . The diagonal element in column $j$ is the positive square root of a random chi-square value with degrees of freedom $n-j$, where $n$ is sample size. Then, using the matrix $A$, the sample covariance matrix $C$ can be obtained such that

$$
C=\left(\frac{1}{n}\right) A \text {. }
$$

The covariance matrix can be rescaled to the sample correlation matrix $R$ such that

$$
R=D^{-\frac{1}{2}} C D^{-\frac{1}{2}}
$$

where $D$ is a diagonal matrix whose elements are the corresponding diagonal entries (i.e., variances) in the covariance matrix $C$.

\section{Computer Program}

Although several computer programs for generating sample correlation matrices without model error are available (e.g., Beasley, 1994; Lee, 1988; Rae, 1997), a program for generating correlation matrices with model error is not easily available. Combining the methods described above, the RANCORR program generates population and sample correlation matrices with model error. That is, the population matrix is generated using the Tucker-Koopman-Linn model and sample matrices are generated using Wijsman's algorithm. RANCORR, written in SAS Interactive Matrix Language, SAS/IML (Version 6.08; SAS Institute, 1992), can be used with either mainframes or personal computers that have the SAS/IML module. Minor modifications may be necessary for some mainframe systems. On a $400 \mathrm{MHz}$ Pentium II personal computer, it takes only $8.94 \mathrm{sec}$ to generate one population correlation matrix and 100 sample matrices.

\section{Numerical Example}

Suppose that the population correlation matrix $P$ is generated on the basis of 10 measured variables, 3 major factors, and 50 minor factors. The data-generation parameters, chosen as an illustration, are presented in Table 1. A unique variance matrix $(D)$, a diagonal matrix, is determined by $D=I-\operatorname{diag}\left(J B J^{\prime}\right)$, where $\operatorname{diag}\left(J B J^{\prime}\right)$ is a diagonal matrix formed from the diagonal elements of $J B J^{\prime}$. In this example, the parameters in matrices $\Phi, Y$, and $\Gamma$ are designed so that the correlation between any two factors is .3. That is, the diagonal and off-diagonal elements of $\Phi$ are 1.0 and .3 , respectively. All elements of $Y$ 
Table 2

Unique Variance Matrix $D$

$D=\left[\begin{array}{cccccccccc}282 & 0 & 0 & 0 & 0 & 0 & 0 & 0 & 0 & 0 \\ 0 & .639 & 0 & 0 & 0 & 0 & 0 & 0 & 0 & 0 \\ 0 & 0 & .529 & 0 & 0 & 0 & 0 & 0 & 0 & 0 \\ 0 & 0 & 0 & .479 & 0 & 0 & 0 & 0 & 0 & 0 \\ 0 & 0 & 0 & 0 & .720 & 0 & 0 & 0 & 0 & 0 \\ 0 & 0 & 0 & 0 & 0 & .342 & 0 & 0 & 0 & 0 \\ 0 & 0 & 0 & 0 & 0 & 0 & .667 & 0 & 0 & 0 \\ 0 & 0 & 0 & 0 & 0 & 0 & 0 & .550 & 0 & 0 \\ 0 & 0 & 0 & 0 & 0 & 0 & 0 & 0 & .659 & 0 \\ 0 & 0 & 0 & 0 & 0 & 0 & 0 & 0 & 0 & .645\end{array}\right]$

are .3. The diagonal and off-diagonal elements of $\Gamma$ are 1.0 and 3 , respectively.

Matrix $W$ is generated using MacCallum and Tucker's (1991) procedure. Minor factor loadings in matrix $W$ are obtained by generating random normal deviates with mean of zero. The standard deviation of these loadings is 1.0 for the first minor factor, and that for each successive minor factor becomes smaller. Thus, the contribution of each preceding minor factor is greater than the successive one. (Because the importance associated with a minor factor decreases in succession, in most cases, 50 minor factors should be enough.) For this purpose, the standard deviation for each successive minor factor is, for example, .8 times the standard deviation for the preceding factor. Loadings for 50 minor factors in the $W$ matrix are then rescaled by rows to satisfy the desired level of contribution of minor factors (i.e., .1) to the variance of the measured variables. The level of model error can be controlled by manipulating the total contribution of minor factors.

Based on the parameters in matrices $J$ and $B$, a unique variance matrix $D$ is determined - that is, by using $D=I$ $-\operatorname{diag}\left(J B J^{\prime}\right)$. Matrix $D$ is presented in Table 2.

Note that the sum of the variance of major, minor, and unique factors should be 1.0 , the variance of a standardized variable. In this example, $10 \%$ and $28.2 \%$ of the variance of the first variable is accounted for by minor and unique factors, respectively, and the remaining variance $(61.8 \%)$ is accounted for by major factors.

The generated population correlation matrix is shown in Table 3. An example of a sample matrix generated on the basis of the population matrix, with a sample size of 400 , is also presented in Table 3 .

\section{The Importance of Including Model Error}

RANCORR generates correlation matrices that include model error as well as sampling error based on the work of MacCallum and Tucker (1991). In their paper, MacCallum and Tucker present model error and sampling error as the major sources of error in factor analysis. Cudeck and Henly (1991) have also distinguished between different types of error that result in lack of fit in structural equation modeling. In their framework, Cudeck and Henly term model error discrepancy of approximation, indicating the lack of fit of the population data to the hypothesized model. The error due to approximation represents the situation in which the model does not fit perfectly to the population.

In most cases, model error (or discrepancy of approximation) exists because no mathematical model accounts

Table 3

Generated Correlation Matrices

\begin{tabular}{lrrrrrrrrrr}
\hline $\begin{array}{l}\text { Population Matrix, } P \\
1.000\end{array}$ & & & & & & & & & \\
.335 & 1.000 & & & & & & & & \\
.398 & .363 & 1.000 & & & & & & & \\
.548 & .272 & .388 & 1.000 & & & & & & \\
.014 & .083 & .028 & -.073 & 1.000 & & & & & \\
.295 & .136 & .100 & .124 & .337 & 1.000 & & & & \\
.009 & .103 & .045 & -.050 & .250 & .348 & 1.000 & & & \\
.209 & .213 & .202 & .114 & .290 & .454 & .290 & 1.000 & & \\
.024 & .069 & .091 & .002 & .053 & .126 & .029 & .142 & 1.000 & \\
-.004 & .051 & .094 & .008 & .031 & .100 & .025 & .143 & .335 & 1.000 \\
Sample Matrix, $R(n=400)$ & & & & & & & \\
1.000 & & & & & & & & & \\
.375 & 1.000 & & & & & & & & \\
.439 & .399 & 1.000 & & & & & & & \\
.579 & .333 & .449 & 1.000 & & & & & & \\
.014 & .086 & .049 & -.051 & 1.000 & & & & & \\
.276 & .148 & .092 & .124 & .353 & 1.000 & & & & \\
.018 & .094 & .058 & -.031 & .249 & .379 & 1.000 & & & \\
.229 & .218 & .198 & .147 & .296 & .454 & .294 & 1.000 & & \\
.035 & .071 & .142 & .020 & .078 & .121 & .002 & .118 & 1.000 & \\
.003 & .083 & .149 & .013 & .100 & .134 & .024 & .145 & .342 & 1.000 \\
\hline
\end{tabular}


exactly for real-world phenomena. In the context of the common-factor model, model error exists because measured variables cannot be accounted for exactly by a small number of common and unique factors. Thus, a typical simulation process of constructing population correlation matrices directly from the common-factor model (without model error) and generating sample correlation matrices from those populations is not appropriate, because the process incorporates only sampling error, ignoring model error, which is another main source of error in factor analysis. Thus, simulated data need to include the numerous minor factors that exist in most real data, in addition to the major factors that provide the basic structure of correlations among the measured variables.

Because RANCORR generates sample correlation matrices from population matrices that include model error, generated correlation matrices do not simply represent the cleanly defined common-factor structure underlying the correlations. Rather, the correlation matrices contain a realistic level of noise. The noise serves to improve generalizability of the results from simulation studies in factor analysis. In spite of the importance of incorporating model error in generating data, only a few studies (e.g., Hakstian, Rogers, \& Cattell, 1982; Humphreys \& Montanelli, 1975; MacCallum \& Tucker, 1991) have used the procedure that incorporates model error. For the generation of realistic data, more frequent use of the procedure is recommended.

\section{Program Availability}

The SAS/IML codes for RANCORR are available by contacting S. Hong, Department of Education, University of California, Santa Barbara, CA 93106. The program also can be transmitted to interested people via the Internet by sending an e-mail message to shong@education. ucsh edu.

\section{REFERENCES}

BEAsLEy, T. M. (1994). CORRMTX: Generating correlated data matrices in SAS/IML. Applied Psychological Measurement, 18, 95.

Cudeck, R., \& Henly, S. J. (1991). Model selection in covariance structures analysis and the "problem" of sample size: A clarification. Psychological Bulletin, 109, 512-519.

Hakstian, A. R., Rogers, W. T., \& Cattell, R. B. (1982). The behavior of number-of-factors rules with simulated data. Multivariate Behavioral Research, 17, 193-219.

Humphreys, L. G., \& Montanelli, R. G. (1975). An investigation of the parallel analysis criterion for determining the number of common factors. Multivariate Behavioral Research, 10, 193-205.

KaISER, H. F., \& Dickman, K. (1962). Sample and population score matrices and sample correlation matrices from an arbitrary population correlation matrix. Psychometrika, 27, 179-182.

LEE, S. (1988). WCORCOV: Generation of sample correlation/covariance matrices. Applied Psychological Measurement, 12, 437.

MacCallum, R. [C.] (1986). Specification searches in covariance structure modeling. Psychological Bulletin, 100, 107-120.

MacCallum, R. C., \& TUCKER, L. R. (1991). Representing sources of error in the common-factor model: Implications for theory and practice. Psychological Bulletin, 109, 502-511.

MCDonald, R. P. (1989). An index of goodness-of-fit based on noncentrality. Journal of Classification, 6, 97-103.

RAE, G. (1997). A FORTRAN 77 program for generating sample correlation matrices. Educational \& Psychological Measurement, 57, 189-192.

SAS Institute (1992). SAS/IML (Release 6.08). [Computer program]. Cary, NC: Author.

Silvia, E. S., \& MacCallum, R. C. (1988). Some factors affecting the success of specification searches in covariance structure modeling. Multivariate Behavioral Research, 23, 297-326.

TUCKER, L. R., KOOPMAN, R. F., \& LiNN, R. L. (1969). Evaluation of factor analytic research procedures by means of simulated correlation matrices. Psychometrika, 34, 421-459.

WIJSMAN, R. A. (1959). Applications of a certain representation of the Wishart matrix. Annals of Mathematical Statistics, 30, 597-601.

(Manuscript received November 3, 1998; revision accepted for publication March 8, 1999.) 